\title{
Control of a Wind Power Plant with a Synchronous Permanent Magnet Generator and a Magnetic Variator
}

\author{
Andrey A. Achitaev*, \\ Konstantin A. Barkhatov and Sergey N. Udalov \\ Siberian Federal University \\ Sayanogorsk, Russian Federation
}

Received 07.02.2021, received in revised form 10.02.2021, accepted 16.02.2021

\begin{abstract}
Currently, the use of wind power plants with magnetic speed reduction is beginning to develop in the world literature. Features of the application of these systems in combination with a wind power plant allows you to solve the issue of its interface with the electric power system. The controlled flexible connection between the turbine and the generator makes it possible to coordinate the control of the load angle of the synchronous generator. In this paper, we consider a developed controller that provides integrated control of a wind turbine with a magnetic variator with a variable pitch of the blade angle and a variable speed of rotation of the wind turbine. A promising direction for the construction of wind power systems is currently the use of electromagnetic variators as part of wind power plants. This work is devoted to the study of electric power systems that include wind turbines with electromagnetic variators. The variator is built between the wind turbine and the generator, instead of a mechanical gearbox. The high-speed change in the torque of the electromagnetic variator allows you to keep the speed of the generator. Due to the inherent non-linearity of the wind turbine and CVT, a set of operational and emergency modes was defined and then the controller was designed for each operating point. In addition, the aerodynamic torque and effective wind speed are estimated online and a planned variable schedule for the controller implementation is obtained. Was tested by simulating with MATLAB/Simulink.
\end{abstract}

Keywords: wind turbine, wind energy conversion systems, CVT, controlled flexible communication.

(C) Siberian Federal University. All rights reserved

This work is licensed under a Creative Commons Attribution-Non Commercial 4.0 International License (CC BY-NC 4.0).

* Corresponding author E-mail address: achitaevaa@gmail.com 


\title{
Управление ветроэнергетической установкой с синхронным генератором на постоянных магнитах и магнитным вариатором
}

\author{
А. А. Ачитаев, К.А. Бархатов, С. Н. Удалов \\ Сибирский федеральный университет \\ Российская Федераџия, Саяногорск
}

\begin{abstract}
Аннотация. В настоящее время в мировой литературе начинает получать развитие применение ветроэнергетических установок с магнитной редукцией скорости. Особенности применения данных систем в сочетании с ветроэнергетической установкой позволяет решить вопрос ее сопряжения с электроэнергетической системой. Управляемая гибкая связь между турбиной и генератором дает возможность согласовать управление углом нагрузки синхронного генератора. В данной работе рассматривается разработанный контроллер, обеспечивающий комплексное управление ветровой турбиной с магнитным вариатором с переменным шагом угла заклинения лопасти и переменной скоростью вращения турбины ВЭУ. Перспективным направлением построения ветроэнергетических систем в настоящее время является применение электромагнитных вариаторов в составе ветроэнергетических установок. Исследованию электроэнергетических систем, в составе которых имеются ветроустановки с электромагнитными вариаторами, посвящена данная работа. Вариатор встраивается между ветровой турбиной и генератором вместо механического редуктора. Быстродействующее изменение вращающего момента электромагнитного вариатора позволяет удерживать частоту вращения генератора. Из-за присущей нелинейности ветровой турбины и вариатора был определен набор эксплуатационных и аварийных режимов, затем контроллер проектировался для каждой рабочей точки. Кроме того, аэродинамический момент и эффективная скорость ветра оцениваются онлайн и получается планируемый график переменной для реализации контроллера. Потенциал метода был проверен путем моделирования с помощью MATLAB/Simulink.
\end{abstract}

Ключевые слова: ветровая турбина с вариаторным изменяемым шагом, ветровая турбина, системы преобразования энергии ветра, вариатор.

Цитирование: Ачитаев, А.А. Управление ветроэнергетической установки с синхронным генератором на постоянных магнитах и магнитным вариатором / А. А. Ачитаев, К. А. Бархатов, С. Н. Удалов // Журн. Сиб. федер. ун-та. Техника и технологии, 2021, 14(1). С. 72-96. DOI: 10.17516/1999-494X-0289

Ветроэнергетические установки (ВЭУ) с вариативным изменяемым шагом ветровых турбин (VS-VP WT) занимают все большую долю на рынке ветроэнергетики. Эта конфигурация является лучшей для мультимегаватных машин, потому что она может не только максимизировать захват энергии в широком диапазоне скоростей ветра, но и уменьшить механическую усталость с использованием системы управления и аэродинамики турбины. В этой конфигурации турбина работает с переменной скоростью вращения и фиксированного шага изменения угла заклинения, если скорость ветра ниже номинальной (рис. 1, зона 2) для достижения максимальной аэродинамической эффективности. Когда скорость ветра находится выше номинальной (зона 3), скорость ветроколеса регулируется в пределах его номинального значения путем изменения на различный угол заклинения лопастей для обеспечения механических ограничений, чтобы они не были превышены. Должен быть разработан контроллер для плавного перехода между двумя регионами, а также для обеспечения других требований во время 
переходного периода, таких как ограничение шума, минимизации колебания выходной мощности и т. д.

Подъемная сила, действующая на лопасти ветроколеса и создающая крутящий момент, является нелинейной функцией в зависимости от скорости ветра, скорости турбины и шага угла заклинения. Кроме того, современные крупногабаритные ветровые турбины обычно оборудованы отдельными приводами для каждой лопасти, датчиками силы, момента или акселерометрами на башне, гондоле, а также они производят вибродиагностику лопастей. Объединить эти входы и выходы можно с помощью структуры комплексного управления ВЭУ.

Базовая структура системы ВЭУ показана на рис. 2. Эта конфигурация имеет два контура, которые работают независимо друг от друга. В верхней части рис. 2 представлен контур управления шагом угла заклинения, который имеет важную роль для регулирования скорости ротора турбины в зоне 3. Когда ветер имеет скорость ниже номинальной, угол заклинения лопасти должен достигать оптимального значения, так как это необходимо для поддержания

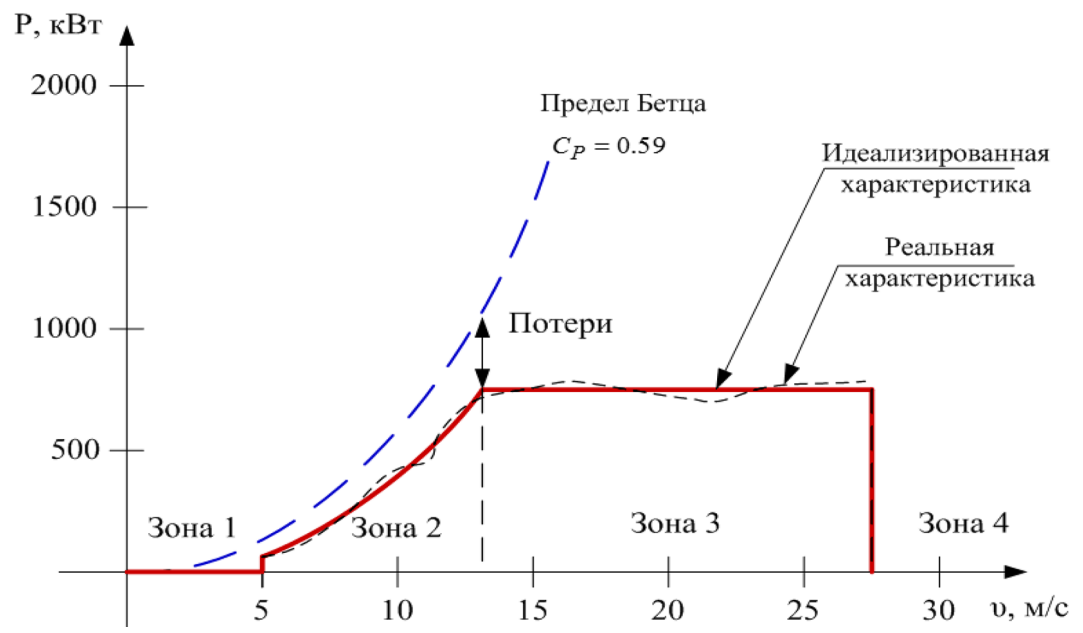

Рис. 1. Энергетическая характеристика ВЭУ

Fig. 1. Energy characteristics of wind turbines

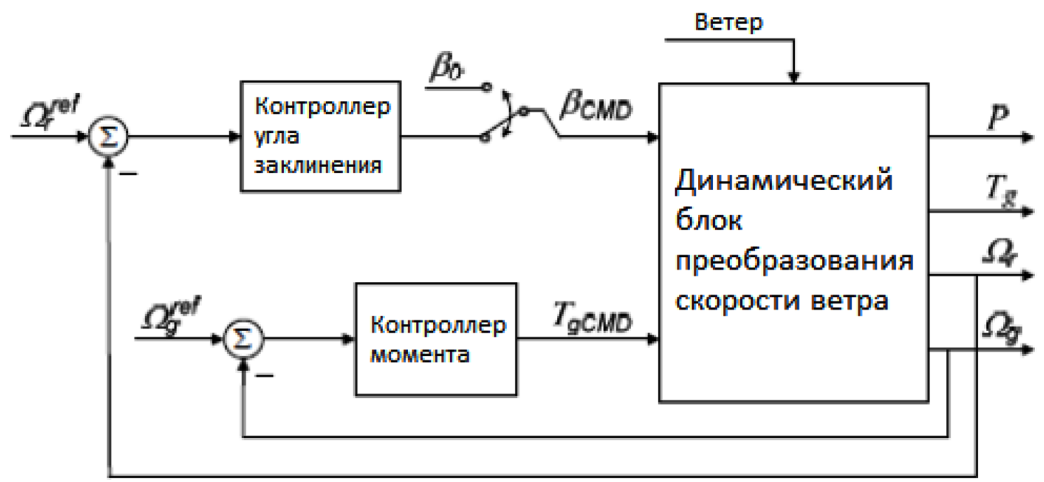

Рис. 2. Структурная схема алгоритма управления мегаваттной ветровой турбиной

Fig. 2. Block diagram of the megawatt wind turbine control algorithm

$$
-74-
$$


работы с максимальным коэффициентом мощности $C_{p}$. Кроме того, в этом регионе различная скорость ротора пропорциональна скорости ветра и является функцией крутящего момента в цикле управления, представленного в нижней части рис. 2.

Алгоритм линейного квадратичного контроля (ЛКК) был применен для ВЭУ и представлен в работах [2-4]. Большинство из них сосредоточили внимание на работу ВЭУ при сильном ветре в зоне 3 или для другого элемента управления со стратегией для разных регионов ветроэнергетической характеристики. Цель этой работы заключается в создании контроллера для ветровой турбины с магнитными вариаторами.

Предлагаемый контроллер имеет ту же стратегию для всех зон эксплуатации. Он синтезирован с целью оптимизации компромисса между максимальным захватом энергии из ветрового потока и смягчения механической нагрузки на узлы ВЭУ. Из-за нелинейности ветровой турбины контроллер предназначен для конкретных оперативных моментов, а именно при изменении скорости ветра, нагрузки и в аварийных режимах.

Основная компоновка ВЭУ с вариатором, работающей с переменной скоростью вращения при преобразовании энергии ветра, показана на рис. 3.

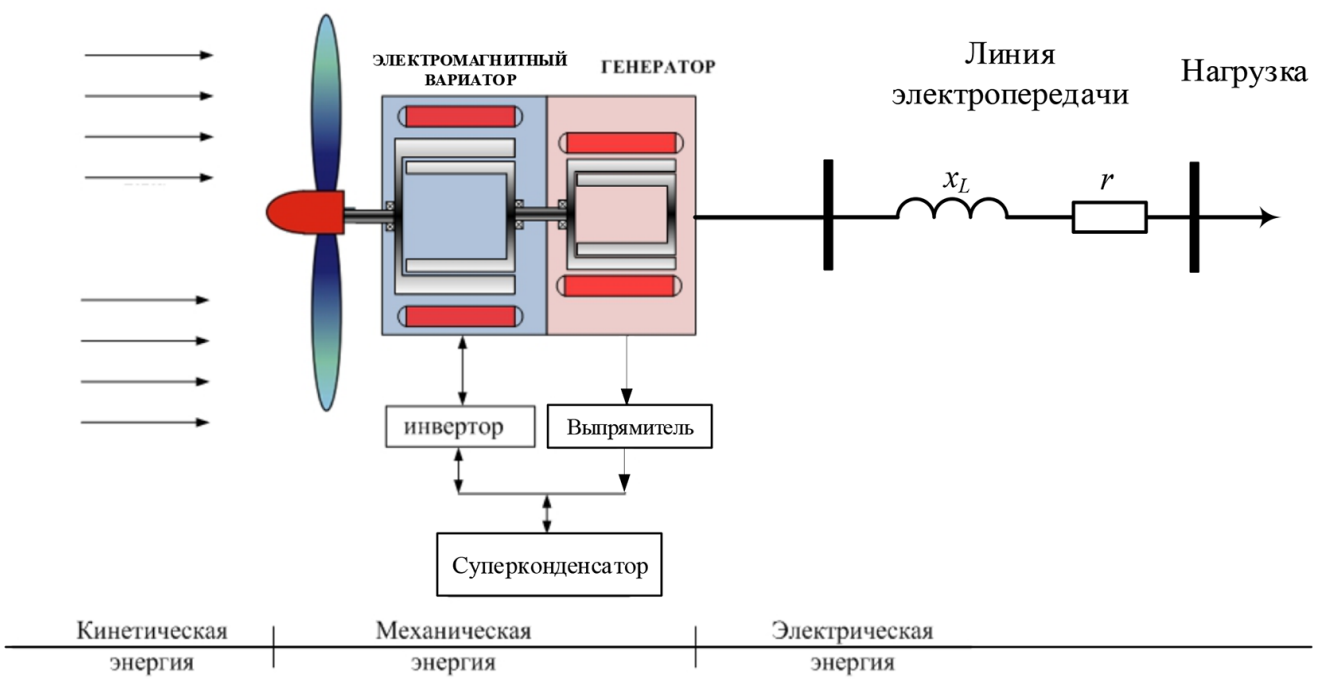

Рис. 3. Компоновка ВЭУ с использованием электромагнитного вариатора

Fig. 3. Layout of a wind turbine using an electromagnetic CVT

Структура электромагнитного вариатора представлена на рис. 4.

\section{Модель ветровой турбины ВЭУ}

Достаточно продолжительное время ветроэнергетические установки работают во второй зоне. Более того, во второй зоне возможно изменение скорости вращения турбины исходя из принципа максимального отбора мощности. Традиционно угол заклинения во второй зоне равен нулю и регулируется только в третей зоне. Однако канал управления углом заклинения можно использовать для управления моментом ветровой турбины. 

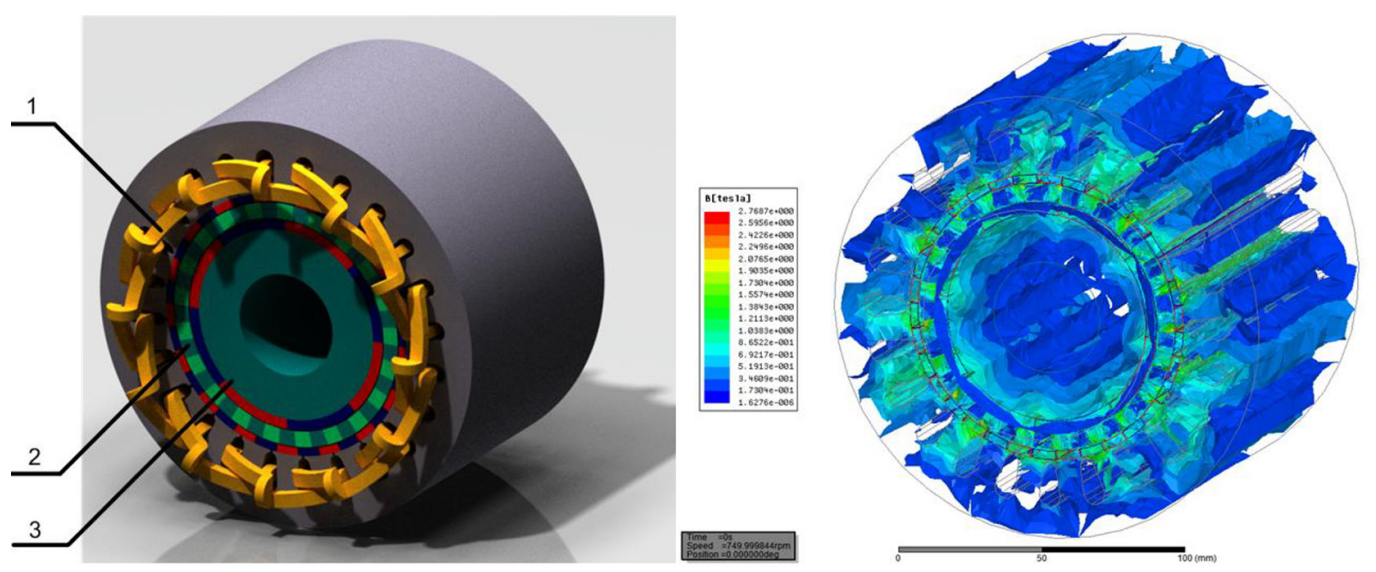

Рис. 4. Электромагнитный вариатор: а - эскиз: 1 - звено управления; 2 - тихоходный ротор с ферромагнитными сегментами; 3 - быстроходный ротор; б - расчет распределения магнитного поля при номинальной нагрузке

Fig. 4. Electromagnetic CVT: a - sketch: 1 - control link; 2 - low-speed rotor with ferromagnetic segments; 3 high-speed rotor; $\mathrm{b}$ - calculation of the magnetic field distribution at rated load

Скорость ветра $-v$ определяется как средняя скорость ветра $v_{m}$ и турбулизированной составляющей $v_{t}$ и представлена как [5]

$$
v=v_{m}+v_{t}
$$

Скорость ветра определяется в плоскости вращения ветроколеса, где $d$ является продольным перемещением гондолы.

$$
v_{r}=\mathrm{v}-d
$$

Подъемная сила, действующая на ротор, создает полезный крутящий момент ветровой турбины, и аэродинамические силы захватывают от энергии ветра мощность, которая описывается системой нелинейных уравнений:

$$
\begin{aligned}
& F_{a}=\frac{1}{2} R^{2} C_{T}(\lambda, \beta) v_{r}, \\
& T_{a}=\frac{1}{2} R^{3} \frac{C_{P}(\lambda, \beta)}{\lambda} v_{r}^{2}, \\
& P_{a}=\frac{1}{2} R^{2} C_{P}(\lambda, \beta) v_{r}^{3},
\end{aligned}
$$

где $\rho$ - плотность воздуха; $R$ - радиус ветроколеса; $C_{T}$ - коэффициент крутящего момента и $C_{P}-$ коэффициент мощности, который представляет эффективность преобразования энергии ветра турбиной. $C_{T}$ и $C_{P}$ являются функцией быстроходности, которая определяется как

$$
\lambda=\frac{R \Omega_{r}}{v_{r}}
$$

Коэффициенты $C_{T}$ и $C_{P}$ очень важны для турбины при проектировании системы управления. Эти характеристики для различных значений быстроходности и угла заклинения проиллюстрированы на рис. 5 и 6. Рисунок 5 показывает, что существует один набор характеристик,

$$
-76-
$$




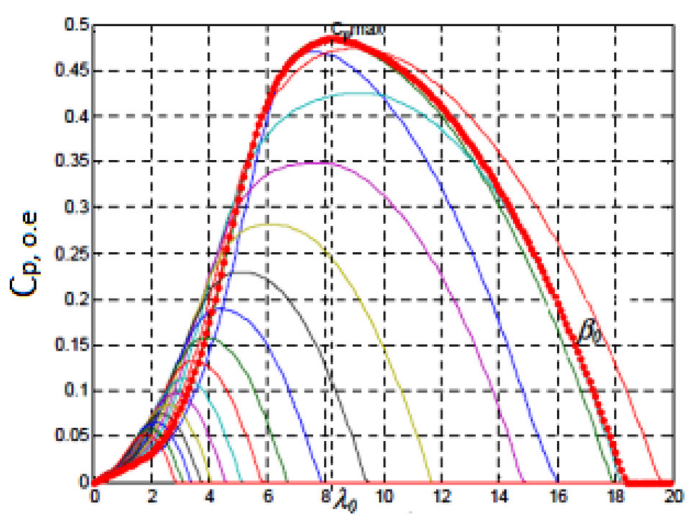

$\lambda$

Рис. 5 Зависимость коэффициента мощности $\mathrm{C}_{\mathrm{P}}$ от $\lambda$

Fig. 5 Dependence of the power factor $C_{P}$ on $\lambda$

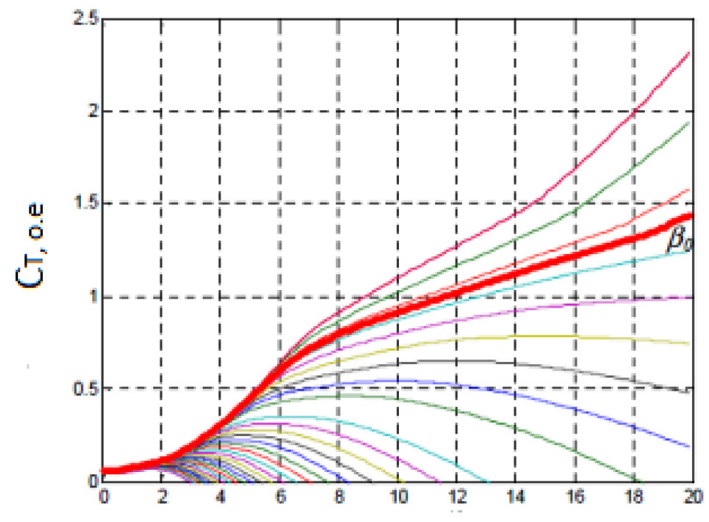

$\lambda$

Рис. 6 Зависимость коэффициента вращающего момента $\mathrm{C}_{\mathrm{T}}$ от $\lambda$

Fig. 6 Dependence of the torque coefficient $\mathrm{C}_{\mathrm{T}}$ on $\lambda$

в котором коэффициент мощности турбины максимален. Это означает, что если угол заклинения фиксируется на оптимальное значение $\beta_{0}$ при различной скорости вращения ветроколеса, которая пропорциональна скорости ветра и при $\lambda_{0}$, где $C_{P}$ максимален, турбина будет извлекать максимальную мощность из ветрового потока.

Однако такое состояние параметров происходит только в зоне 2, в регионах с низкими скоростями ветра. Когда высокая скорость ветра, быстроходность и угол заклинения находятся вдали от их оптимальных значений для обеспечения максимальной мощности и скорость ветроколеса регулируется вокруг их номинальных значений для того, чтобы избежать превышения выработки электрической энергии, а также механических напряжений в узлах ВЭУ.

\section{Модель вариатора}

Электромагнитный вариатор представляет собой электромеханическую систему с изменяемым выходным вращающим моментом, который формирует добавочный момент для стабилизации скорости на валу генератора.

Электромагнитный вариатор - двухмассовая электромеханическая система, которая складывается за счет упругой магнитной связи между турбиной и генератором. Вместе с тем электромагнитный вариатор выполняет функцию демпфера в энергосистеме при переходных процессах в ней.

Для иллюстрации дополнительного приведенного угла рассогласования между турбиной и генератором в электромагнитном вариаторе представлен рис. 7, где изображены сравнительные механические характеристики между классической компоновкой (а) и компоновкой с электромагнитным вариатором (б). Классическая компоновка имеет одно колебательное звено, выраженное электрическим углом $\delta$ между векторами ЭДС генератора и напряжением ШБМ. В компоновке с электромагнитным вариатором отражено два колебательных звена, в ней можно наблюдать влияние дополнительного приведенного угла рассогласования в двухмассовой системе, формирующее жесткость между турбиной и генератором. 


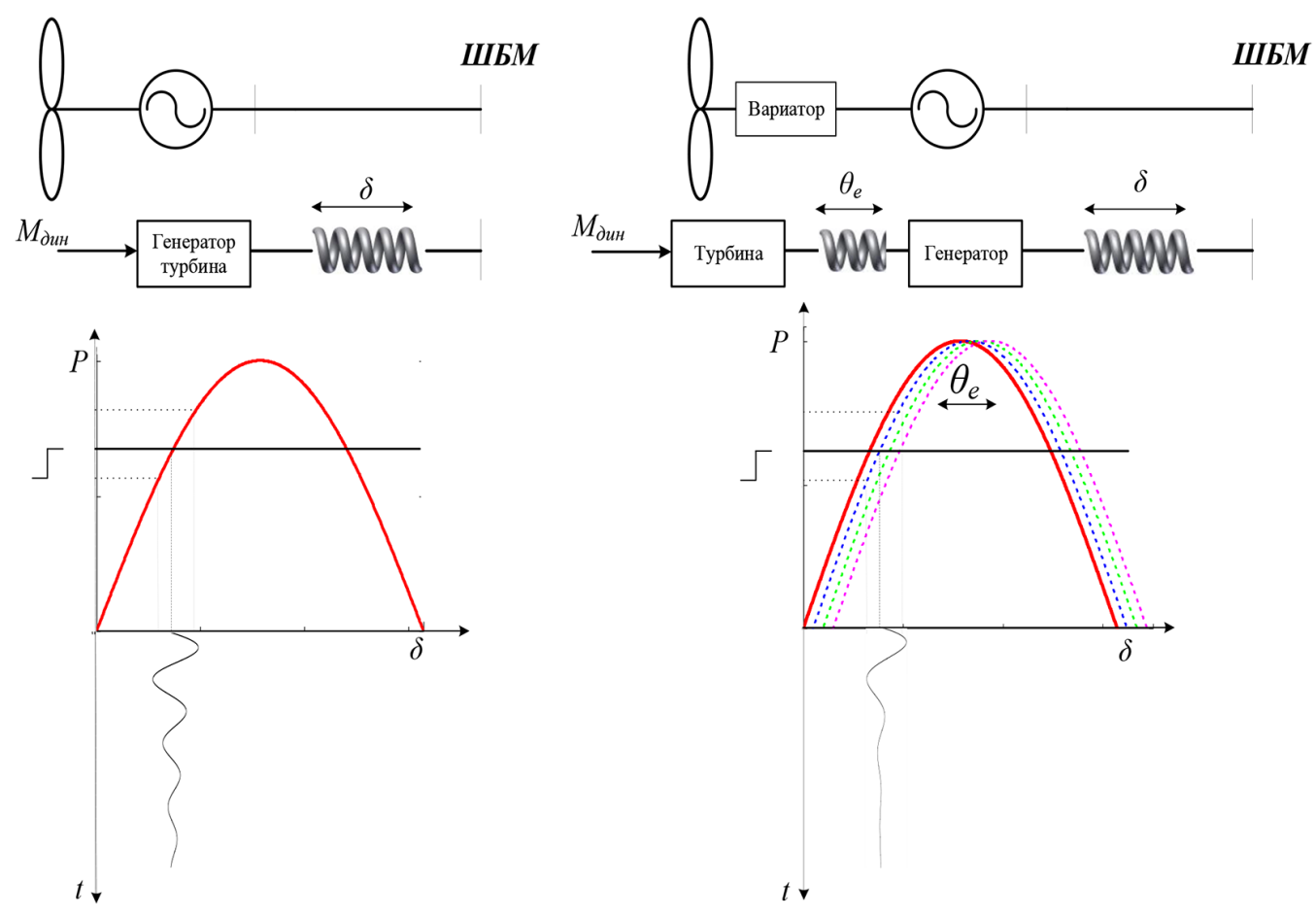

Рис. 7. Сравнительная механическая характеристика: а - системы турбина-генератор-линия-ШБМ; б системы турбина-вариатор-генератор-линия-ШБМ

Fig. 7. Comparative mechanical characteristics: a - turbine-generator-line-IPB systems; $b$ - turbine-variatorgenerator-line-IPB systems

Аэродинамический момент из уравнения (4) является входной величиной для вариатора со стороны оси низкой скорости. Редуктор может моделироваться как два инерционных звена двухмассовой системы, соединенные пружиной, с одной стороны турбина, с другой - редуктор (рис. 8).

На высокоскоростной оси вариатора и генератора установлены датчики, дающие возможность контролировать реакцию крутящего момента от генератора. Динамическая модель редуктора моделируется исходя из выражений (7) [6].

$$
\left\{\begin{array}{l}
J_{h} \frac{d \omega_{h}}{d t}=M_{e m}-\frac{M_{\max }}{G_{r}} \cdot\left[\sin \left(p_{h} \theta_{h}-n_{s} \theta_{\text {Load }}\right)\right]-B_{h} \omega_{h}-\mu_{a p}\left(p_{h} \omega_{h}-n_{s} \omega_{\text {Load }}\right) \\
J \frac{d \omega_{0}}{d t}=M_{\max } \sin \left(p_{h} \theta_{h}-n_{s} \theta_{\text {Load }}\right)-M_{\text {Turbine }}-B_{0} \omega_{0}-\mu_{a p}\left(p_{h} \omega_{h}-n_{s} \omega_{\text {Load }}\right),
\end{array}\right.
$$

где $J_{h}, B_{h}$ - момент инерции и вязкое затухание ротора высокой скорости; $\omega_{0}, J, B_{0}-$ угловая скорость, суммарный момент инерции тихоходного вала, коэффициент вязкого трения; $\mu_{a p}-$ коэффициент затухания; $n_{s}$ - число ферромагнитных модуляторов; $p_{h}$ - число пар полюсов быстроходного ротора; $G_{r}=n_{s} / p_{h}-$ передаточное отношение магнитной трансмиссии; $M_{\text {Turbine }}-$ момент турбины ВЭУ.

Двухмассовая электромеханическая система представляет собой наиболее удобную модель для изучения влияния упругих механических связей. На первую массу с моментом инер- 


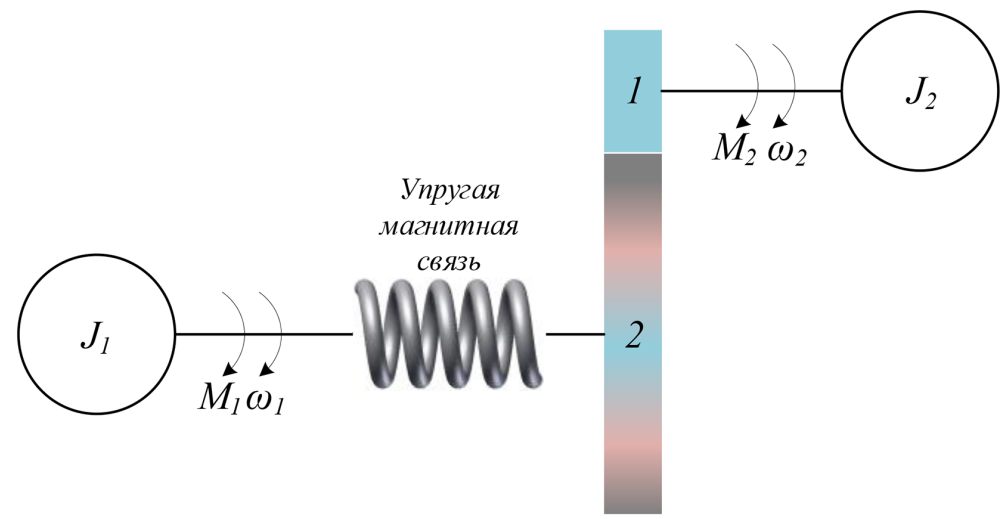

Рис. 8. Схема двухмассовой электромеханической системы

Fig. 8. Diagram of a two-mass electromechanical system

ции $J_{l}$ действует приложенный к ней движущий момент турбины $M_{l}=M_{m y р б}$, которому противодействует момент упругого закручивания $M_{2}$ между первой и второй массами, обусловленный упругостью магнитного поля в воздушном зазоре магнитного редуктора. Движущим моментом, действующим на вторую массу, является момент упругого закручивания, а момент сопротивления определяется как приведенный к ведомому валу редуктора момент сопротивления движению, создаваемый рабочим механизмом - $M_{C}$. В результате можно представить следующее математическое описание движения рассматриваемой двухмассовой механической системы электропривода:

$$
\left\{\begin{array}{l}
M_{1}-M_{3}=J_{1} \frac{d \omega_{h}}{d t}, M_{2}-M_{C}=J_{2} \frac{d \omega_{2}}{d t} \\
M_{3}=M_{2} / G, M_{2}=f(\theta)=M_{m} \sin \theta \\
\theta_{e}=\varphi_{h}-\varphi_{2}=\int\left(p_{h} \omega_{h}-p_{2} \omega_{2}\right) d t
\end{array}\right.
$$

где $G=p_{1} / p_{2}-$ передаточный коэффициент.

На рис. 9 представлена структурная схема, соответствующая системе уравнений (8). Она демонстрирует возможность моделировать динамические процессы магнитного редуктора при различных углах рассогласования, так как в ней учтена тригонометрическая связь между входным и выходным моментами.

Структурная схема, приведенная на рис. 9, содержит нелинейное звено. Для анализа таких схем можно использовать аппаратный метод детализированных структурных схем, получающий в последнее время все более широкое распространение. Его использование позволяет выделять линейные и нелинейные области модели, преобразовывать линейную часть по известным правилам [7], формировать каналы вычисления мгновенных значений мощности и электромагнитных усилий, наращивать дополнительными элементами в соответствии с расширением реальной электромеханической системы.

Как показано в [8], метод является универсальным и позволяет изменять модели отдельных элементов без изменения структуры модели системы в целом. 


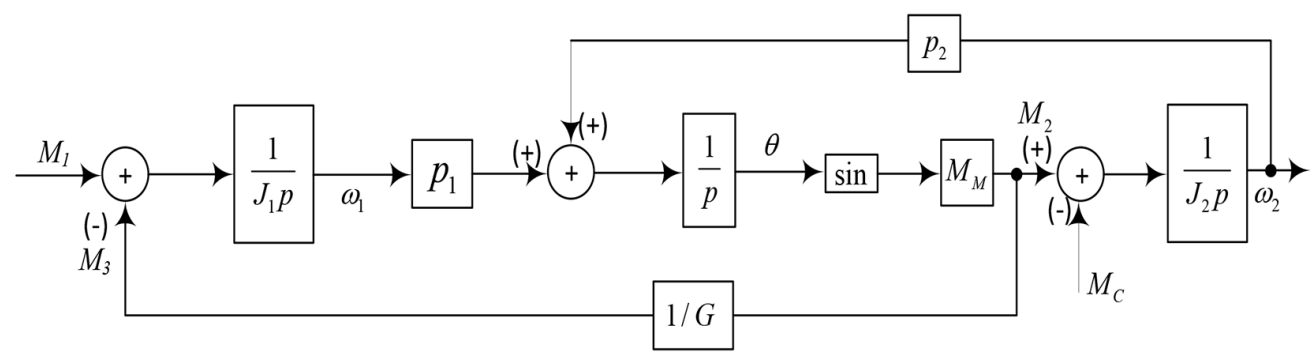

Рис. 9. Структурная модель магнитного редуктора

Fig. 9. Structural model of a magnetic gearbox

Отличие вариатора от магнитного редуктора состоит в том, что он имеет внешний ротор управления. Следовательно, изменяя скорость внешнего ротора (ротора управления) $\omega_{2}$ в соотношении к входной скорости ротора $\omega_{3}$, передаточное отношение между входным и выходным ротором может регулироваться. Моменты $M_{2}, M_{k}$ и $M_{3}$, действующие на роторы соответственно, могут быть получены при условии пренебрежения потерями. Тогда можно записать:

$$
\begin{aligned}
& M_{2}+M_{k}+M_{3}=0 ; \\
& \omega_{2} M_{2}+\omega_{k} M_{k}+\omega_{2} M_{3}=0 .
\end{aligned}
$$

На основании этого получается момент на быстроходном валу:

$$
M_{k}=p_{k} M_{m} \sin \left(p_{k} \varphi_{k}-p_{2} \varphi_{2}-p_{3} \varphi_{3}\right)
$$

Примем в качестве допущения в уравнениях (10) отсутствие потерь в магнитной системе

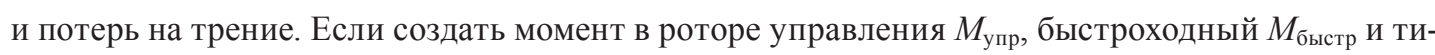
хоходный вал $M_{\text {тих }}$ соответственно изменят свои значения, тогда система уравнений динамики электромеханического преобразователя будет иметь вид [9]

$$
\left\{\begin{array}{c}
J_{\text {упр }} \frac{d^{2} \varphi_{2}}{d t^{2}}=M_{\text {упр }}-p_{2} M_{m} \sin \left(p_{k} \varphi_{k}-p_{2} \varphi_{2}-p_{3} \varphi_{3}\right) \\
J_{\text {быстр }} \frac{d^{2} \varphi_{3}}{d t^{2}}=M_{\text {быстр }}-p_{3} M_{m} \sin \left(p_{k} \varphi_{k}-p_{2} \varphi_{2}-p_{3} \varphi_{3}\right) \\
J_{\text {тих }} \frac{d^{2} \varphi_{k}}{d t^{2}}=M_{\text {тих }}+p_{k} M_{m} \sin \left(p_{k} \varphi_{k}-p_{2} \varphi_{2}-p_{3} \varphi_{3}\right)
\end{array}\right\}
$$

где $J_{\text {тих }}, J_{\text {быстр, }} J_{\text {упр }}$ - момент инерции роторов низкой, быстрой скорости и ротора управления соответственно.

\section{Динамика башни}

Для учета динамики башни при проектировании контроллера была использована достаточно упрощенная модель асимметричной башни [10]. В этой статье учитывается верхнее смещение башни, соответствующее только первому изгибу в продольном направлении с учетом общей массы гондолы.

В качестве допущения принимается, что выше первого изгиба вращение верхней кромки башни, деформации кручения и эффекты рыскания игнорируются. Режим передней кромки в продольном направлении первого изгиба описывается следующим выражением: 


$$
M_{T} \ddot{d}+C_{T} \dot{d}+K_{T} d-F_{a}=0,
$$

где $M_{T}$ - эффективная масса верхней части башни, которая определяется как сумма массы гондолы, включая турбину, плюс эквивалентная масса башни выше первого изгиба $u K_{T}$ соответственно, демпфирование структуры башни и жесткость на изгиб; $d, \ddot{d}$ и продольное перемещение, скорость и ускорение соответственно верхней передней кромки башни.

\section{Динамика генератора}

Наиболее распространенные типы генераторов для ветровой турбины - асинхронизированые генераторы двойного питания (АГДП) и синхронные генераторы на постоянных магнитах (СГПМ) [5].

Крутящий момент генератора контролируется электромагнитным преобразователем частоты - вариатором в случае использования СГПМ, с помощью которого возможно также управлять активной и реактивной мощностью генератора.

Известно, что СГПМ можно описать следующей системой уравнений [11]:

$$
\left\{\begin{array}{l}
U_{d}=r i_{d}+L_{d} \frac{d i_{d}}{d t}-L_{q} i_{q} \frac{d \delta_{\ni}}{d t}, \\
U_{q}=r i_{q}+L_{q} \frac{d i_{q}}{d t}+\left(L_{d} i_{d}+k_{0} W p_{n} \Phi_{f}\right) \frac{d \delta_{\ni}}{d t}, \\
J \frac{d^{2} \delta_{\ni}}{d t^{2}}=\frac{3}{2}\left[\left(L_{d}-L_{q}\right) i_{q} i_{d}+k_{0} W p_{n} \Phi_{f} i_{q}\right]-M_{c},
\end{array}\right.
$$

где $U_{d}, U_{q}, i_{d}, i_{q}, L_{d}, L_{q}$ - составляющие напряжения, тока и индуктивности по продольной и поперечной осями соответственно; $\delta_{э}$ - электрический угол, характеризующий положение ротора; $r$ - активное сопротивление статора; $\Phi_{f}-$ магнитный поток на полюс; $p_{\text {п }}$ - число пар полюсов; $W$ - число витков; $k_{0}$ - обмоточный коэффициент; $J$ - момент инерции; приведенный к валу; $M_{\mathrm{c}}$ - момент сопротивления.

Структурная модель СГПМ, соответствующая этой системе уравнений, представлена на рис. 10.

С учетом упругости магнитного редуктора уравнение механического равновесия запишется как уравнение (13).

Передаточная функция, выражающая отношение между моментом электромагнитного вариатора, скоростью вращения турбины и скоростью вращения генератора, выражена следующими уравнениями [11]:

$$
\begin{aligned}
& \frac{\omega_{0}(p)}{M_{E M}(p)}=\frac{p^{2}+\omega_{a}^{2}}{J_{h} p^{3}+J_{h} \omega_{r}^{2} p} \\
& \frac{\omega_{h}(p)}{M_{E M}(p)}=\frac{\omega_{a}^{2}}{J_{h} p^{3}+J_{h} \omega_{r}^{2} p},
\end{aligned}
$$

где $\omega_{a}=\sqrt{K_{e m} / J}-$ антирезонансная частота; $\omega_{0}$ - скорость турбины; $\omega_{r}=\omega_{0} \sqrt{(R+1)}-$ резонансная частота; $J_{h}$ - момент инерции ротора высокой скорости; $\omega_{h}-$ скорость быстроходного вала; $M_{E M}$ - электромагнитный момент машины. 


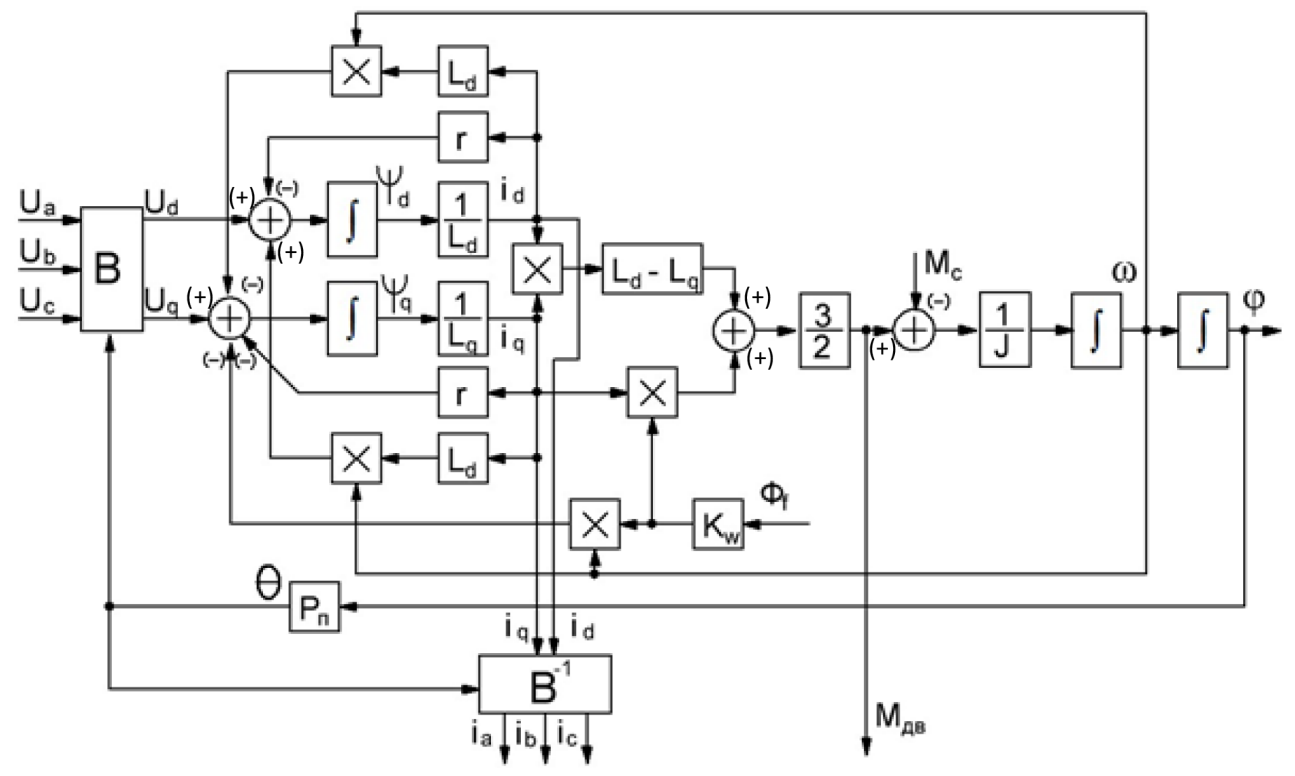

Рис. 10. Структурная модель СГПМ

Fig. 10. Structural model of the SGPM

\section{Привод угла заклинения турбинь}

Пока ветровая турбина работает в зоне 3 , т. е. с большими скоростями ветра, высокая скорость ротора может причинить механическое повреждение и больше не может управляться путем максимального захвата энергии из ветрового потока, потому что это приведет к перегрузке генератора [4]. Как видно на рис. 5, Ср может быть уменьшен путем изменения угла заклинения $\beta$ для поддержания выходной мощности и скорости вращения ветровой турбины, равной номинальному значению, вместо изменения частоты вращения ротора пропорционально скорости ветра, как это делается в зоне 2.

Привод угла заклинения представляет собой нелинейный усилитель, который обычно вращает все лопасти или их часть в унисон. В замкнутом цикле привод может быть смоделирован как линейная динамическая система низкого порядка с насыщением по амплитуде и производной от выходного сигнала. На рис. 11 показана структурная схема модели привода второго

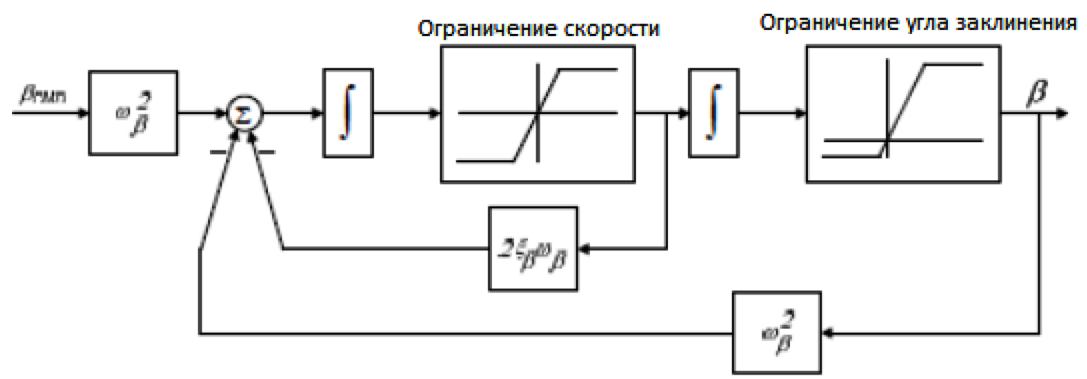

Рис. 11. Модель привода изменения шага угла заклинения второго порядка

Fig. 11. Model of the drive for changing the pitch of the second-order incantation angle

$$
-82-
$$


порядка. Динамическое изменение привода угла заклинения происходит внутри его линейной области и описывается следующей передаточной функцией:

$$
\frac{\beta(s)}{\beta_{C M D}(s)}=\frac{\omega_{\beta}^{2}}{s^{2}+2 \xi_{\beta} \omega_{\beta}+\omega_{\beta}^{2}},
$$

где $\beta$ и $\beta_{C м D}$ являются фактическим и требуемым углом заклинения соответственно; $\omega_{\beta}$ и $\xi_{\beta}$ - соответственно собственная частота и коэффициент демпфирования динамики привода шага. Для мультимегаваттной турбины угол заклинения колеблется от 3 до $90^{\circ}$ и варьируется с максимальной скоростью $\pm 10 \%$.

\section{Контроллер}

\section{A. Модель управления контроллера}

Магнитный вариатор можно представить в виде магнитного редуктора и интегрированного с ним синхронного двигателя, с помощью которого формируется дополнительный вращающий момент, который компенсирует приращение момента нагрузки, чтобы обеспечить стабилизацию скорости вращения или слежение за фазой ведущего генератора.

Эффективное управление синхронного двигателя с постоянными магнитами осуществляется с помощью системы векторного управления. Один из вариантов построения такой системы приведен на рис. 12.

При управлении синхронным двигателем с помощью системы векторного управления (СВУ) достигается настолько высокое быстродействие при формировании момента, что инерционными свойствами этой системы можно пренебречь.

Представление системы векторного управления безынерционным звеном существенно упрощает рассматриваемую систему, структурная схема которой изображена на рис. 13. Особенностью магнитного вариатора является наличие упругой связи между ветровой турбины и гене-

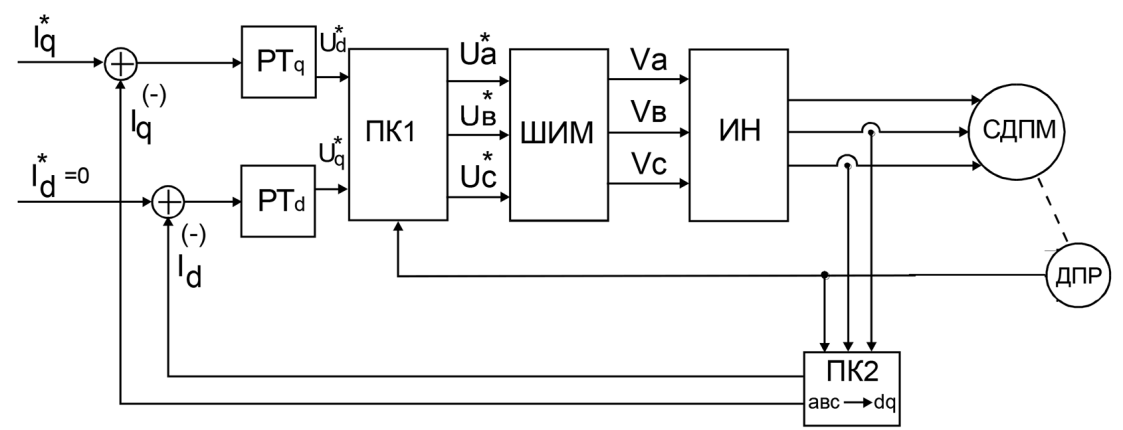

Рис. 12. Функциональная схема системы управления электромагнитным вариатором с пофазной модуляцией и с регулированием токов в системе координат $d$ и $q$ : ПК1, ПК2 - преобразователи координат; ШИМ - широтно-импульсный модулятор; ИН - инвертор напряжения; ДПР - датчик положения ротора; $\mathrm{PT}_{\mathrm{d}}, \mathrm{PT}_{\mathrm{q}}$ - регулятор тока по оси $\mathrm{d}$ и q соответственно; СДПМ - интегрированный синхронный двигатель управления с постоянными магнитами

Fig. 12. Functional diagram of the control system of an electromagnetic variator with phase-by-phase modulation and current regulation in the coordinate system $d$ and $q$ : PK1, PK2-coordinate converters; PWM - pulse-width modulator; IN - voltage inverter; DPR - rotor position sensor; RTd, RTq - current regulator on the $\mathrm{d}$ and q axes, respectively; SDPM - integrated synchronous control motor with permanent magnets

$$
-83-
$$




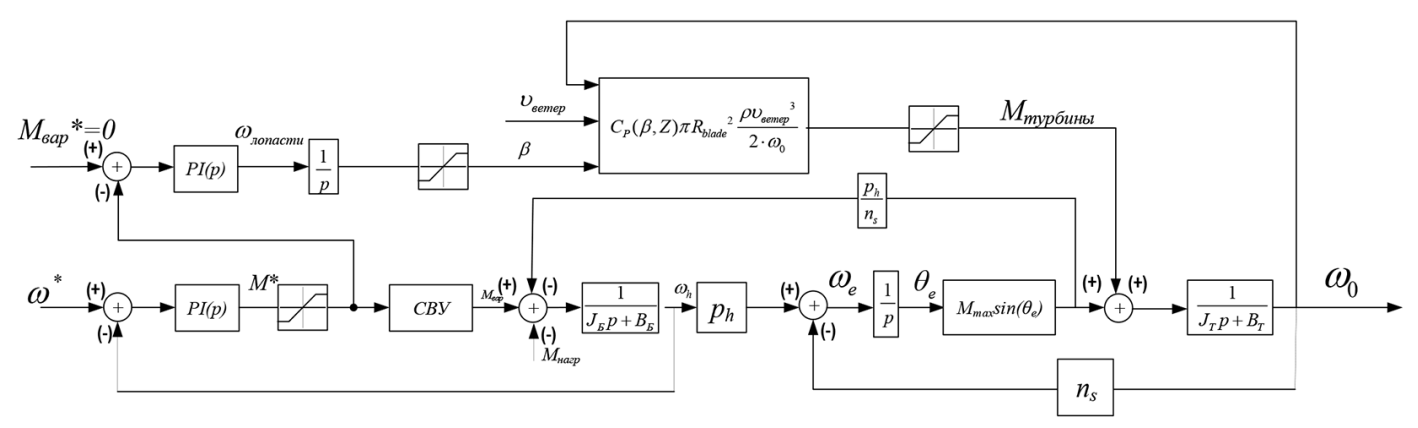

Рис. 13. Структура алгоритма стабилизации скорости вращения быстроходного ротора магнитного вариатора со стороны электрического генератора

Fig. 13 Structure of the algorithm for stabilizing the rotation speed of a high-speed rotor of a magnetic variator on the side of an electric generator

ратором, который приводит к ярко выраженному двухмассовому характеру исследуемой системы, что усложняет синтез регуляторов, обеспечивающих оптимальные переходные процессы.

Существует ряд возможных методик выбора коэффициентов усиления ПИ-регуляторов в зависимости от поставленной задачи. Был применен метод Мэйсона, суть которого состоит в выборе параметров усиления в замкнутой системе при условии, что затухание будут составлять -20 дБ/дек [12-16]. С помощью метода Мэйсона формируется соотношение между текущей скоростью вращения быстроходного вала электромагнитного вариатора и заданной скоростью, которая обеспечивает затухание в - 20 дБ/дек. С использованием метода нулей и полюсов передаточной функции Мэйсона формируется выбор показателей усиления [11].

Синтез каждого контура в системе подчиненного регулирования базируется на идее компенсации основной инерционности объекта управления этого контура последовательным корректирующим звеном с помощью его обратной модели, а для ограничения полосы пропускания в каждом контуре вводится малая некомпенсированная инерционность. Такое построение регулятора всей системы обеспечивает совпадение теоретических и практических результатов только при отработке задающих воздействий при нулевых начальных условиях. В случае наличия ненулевых начальных условий и действия возмущений в объекте процессы во всей системе будут существенно отличаться от оптимальных рассчитанных.

Передаточная функция замкнутой системы управления электромагнитного вариатора равна отношению начальной скорости вращения турбины к заданной скорости турбины и определяется следующим выражением:

$$
\frac{\omega_{0}(p)}{\omega_{r e f}(p)}=\frac{K_{I} \omega_{a}^{2}}{J_{h} p^{4}+K_{P} p^{3}+\left(J_{h} \omega_{0}^{2}(1+R)+K_{I}\right) p^{2}+K_{P} \omega_{a}^{2} p+K_{I} \omega_{a}^{2}},
$$

где $\omega_{a}=\sqrt{K_{e m} / J}$ - антирезонансная частота; $R=J / J_{h}-$ соотношение моментов инерции роторов турбины и генератора; $\omega_{r}=\omega_{0} \sqrt{(R+1)}-$ резонансная частота; $K_{I}, K_{P}-$ коэффициенты ПИ регулятора; $\omega_{0}-$ скорость турбины; $\omega_{\text {ref }}$-заданная скорость вращения.

И, соответственно, передаточная функция равна отношению начальной скорости вращения замкнутой системы к заданной скорости ротора электрического генератора и определяется следующим выражением: 


$$
\frac{\omega_{h}(p)}{\omega_{\text {ref }}(p)}=\frac{\left(K_{I} p^{2}+K_{I} K / J\right)}{J_{h} p^{4}+K_{P} p^{3}+\left(K_{I}+K\left(1+J_{h} / K_{L}\right)\right) p^{2}+K_{P} K / J_{h} \cdot p+K_{I} K / J_{h}}
$$

где $K$ - линеаризованный коэффициент упругости магнитной связи.

Синтез всей системы начинается с построения регулятора для самой быстрой координаты. На рис. 13 отображен комплексный алгоритм стабилизации частоты вращения электрического генератора с помощью магнитного вариатора и аэродинамического контура с регулируемым углом заклинения.

На рис. 14 представлены логарифмические амплитудно-частотные характеристики для магнитного вариатора со стороны генератора и ветроэнергетической турбины, на котором можно явно увидеть антирезонансную и резонансную частоту.

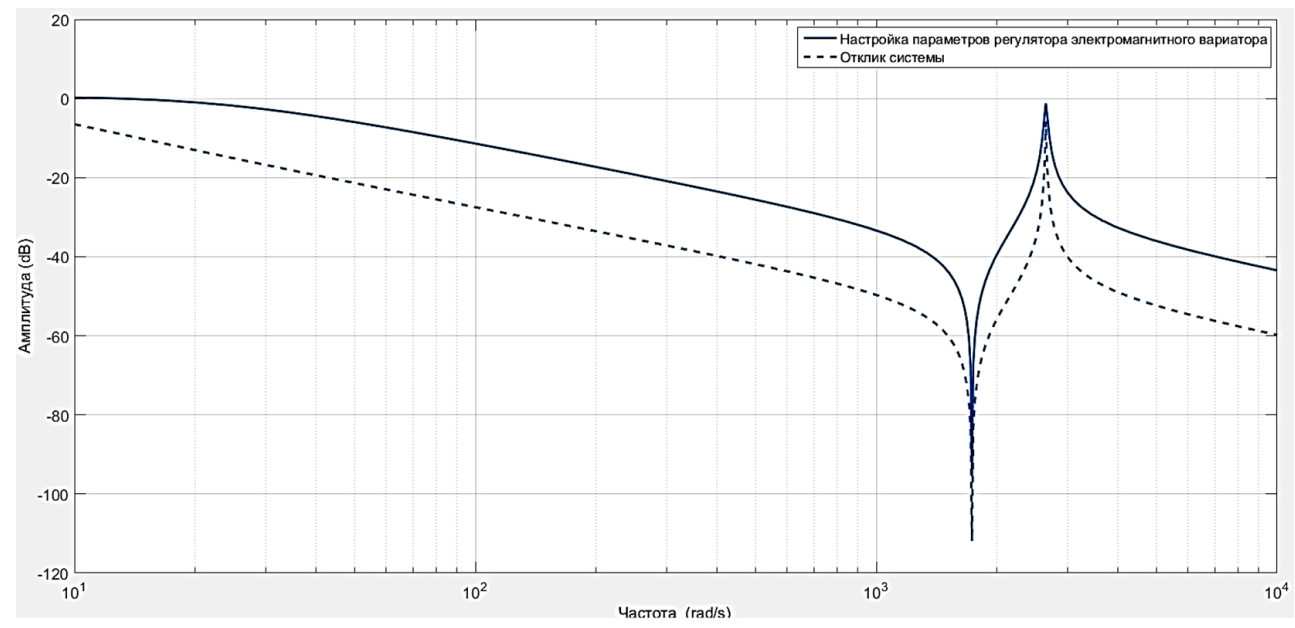

a

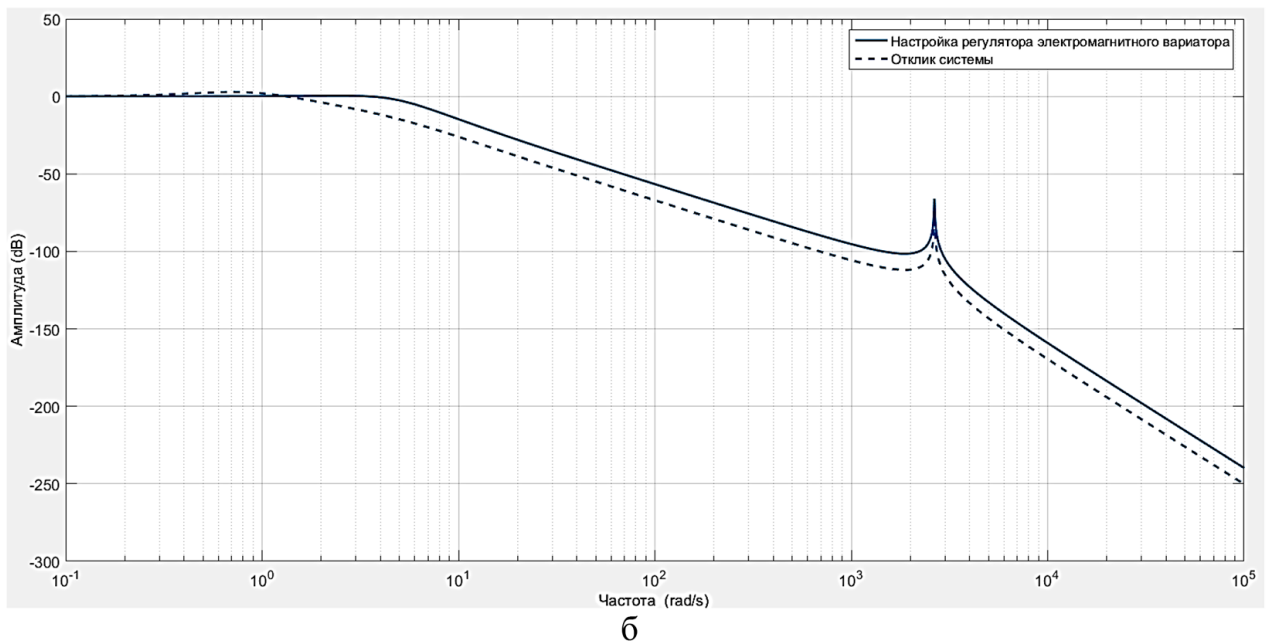

Рис. 14. Расчетная логарифмическая амплитудно-частотная характеристика разомкнутой двухмассовой системы модели: а - электромагнитного вариатора; б - со стороны ветроэнергетической турбины

Fig. 14. Calculated logarithmic amplitude-frequency response of the open two-mass system of the model: a electromagnetic variator; $b$ - from the side of the wind turbine 
Нами представлены исследования по формированию законов и построению алгоритмов комплексного управления частотой вращения электромагнитного вариатора. Для определения показатели качества переходного процесса при работе электромагнитного вариатора в АвЭС проведем синтез законов управления по методу Мейсона.

Значения коэффициента усиления и параметры фильтров зависят от выбранного способа коррекции быстрых движений. Согласно общей методике постоянная времени подсистемы быстрых движений должна быть примерно на порядок меньше постоянной времени медленных движений. Вопросы синтеза системы управления магнитного вариатора с учетом нелинейной упругой связи, вносимой магнитным вариатором, рассмотрены в работах $[16,17]$, в которых система оптимизируется по критерию интегральной ошибки по времени.

Использование аэродинамического управления путем изменения угла заклинения лопасти, плазменного или струйного привода, применение флэпс-устройств дают возможность обеспечить компенсацию вращающего момента и тем самым снизить нагрузку на накопитель энергии быстродействующего контура электромагнитного вариатора. Таким образом, возможно построение тандемного способа поддержания скорости вращения генератора ВЭУ, которое заключается в использовании быстродействующего контура управления через магнитный вариатор, затем действие контура регулирования угла заклинения лопастей позволяет обеспечить стабилизацию скорости вращения синхронных генераторов с постоянными магнитами и, соответственно, напряжения. Необходимо провести математическое описание магнитного вариатора и его динамической модели для дальнейшего синтеза регуляторов, входящих в систему поддержания заданной скорости вращения или фазы положения ротора генератора.

На рис. 15 представлена осциллограмма переходных процессов в звене управления магнитным вариатором при формировании дополнительного момента при последовательном скачкообразном увеличении нагрузки на 20 \%. Сплошной график показывает работу системы без использования контура регулирования угла заклинения, и момент образуется только действием магнитного вариатора. Пунктирный график отображает систему с учетом использования

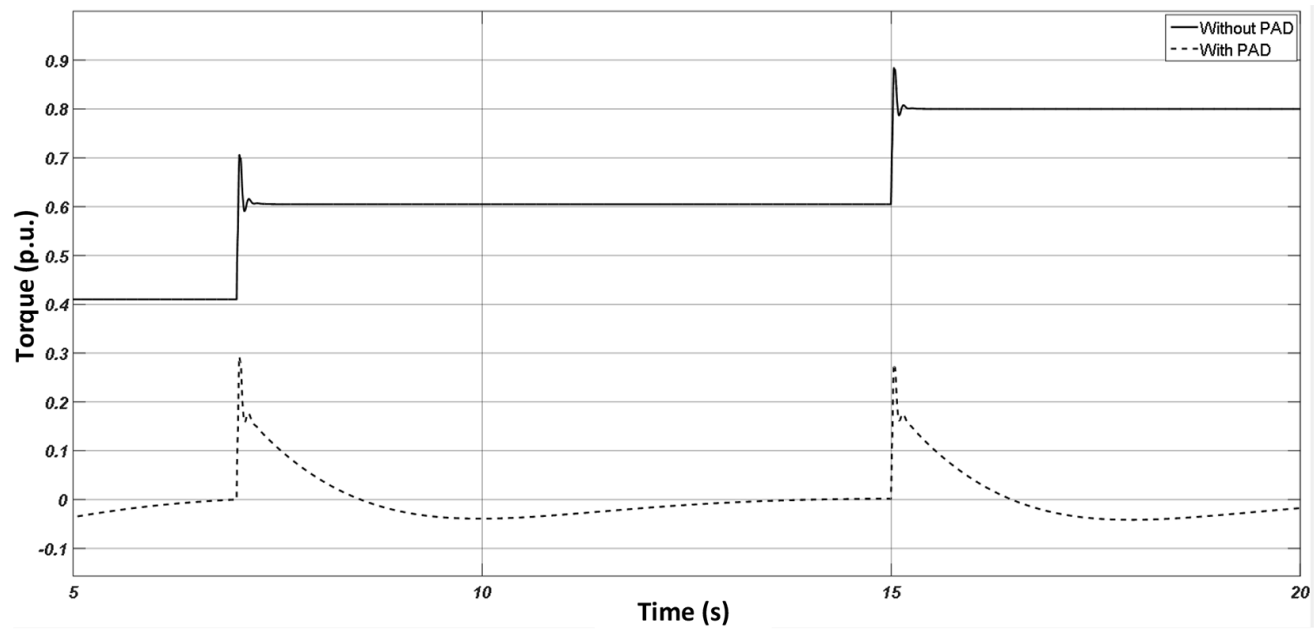

Рис. 15. Осциллограмма момента магнитного вариатора с учетом комплексного управления и без него

Fig. 15. Oscillogram of the moment of the magnetic CVT with and without integrated control

$$
-86-
$$


контура регулирования угла заклинения и контура регулирования момента магнитного вариaтора.

В подавляющем большинстве случаев энергосистемы являются смешанными, т. е. имеют в своем составе электростанции и ветроустановки разных типов.

На рис. 16 изображена структура алгоритма компенсации угла нагрузки магнитным вариатором по ведущему генератору в системе в целях исключения асинхронного хода. Она представляет собой использование дополнительного контура фазовой стабилизации, которая дополняет алгоритм стабилизации скорости регулятором фазового положения СГПМ ВЭУ. Регулирование фазового положения ротора СГ осуществляется с помощью магнитного вариатора со стороны электрического генератора.

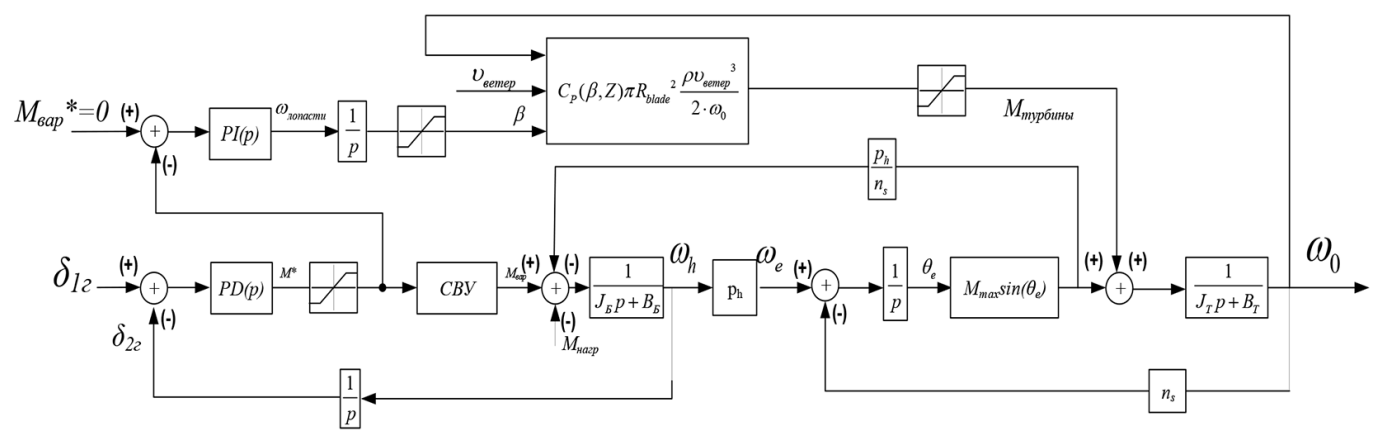

Рис. 16. Структурная схема алгоритма управления электромагнитным вариатором с фазовой коррекцией Fig. 16. Block diagram of the control algorithm for the electromagnetic CVT with phase correction

Передаточная функция контура управления фазовой коррекцией электромагнитного вариатора ведомого генератора определяется отношением начального фазового положения ротора ведомого генератора к заданному углу положения ведущего генератора в АвЭС и соответствует следующему выражению:

$$
\frac{\theta_{\text {angle }}(p)}{\theta_{\text {ref }}(p)}=\frac{K_{P}\left(p^{2}+\omega_{a}^{2}\right)}{J_{h} p^{4}+K_{D} p^{3}+\left(J_{h} \omega_{a}^{2}(1+R)+K_{P}\right) p^{2}+K_{D} \omega_{a}^{2} p+K_{P} \omega_{a}^{2}}
$$

где $K_{D}$, $K_{P}$ - коэффициенты пропорционально-дифференциального регулятора.

На рис. 16 отображена структурная схема алгоритма фазовой коррекции, в которой также реализовано комплексное управление моментом ветровой турбины через угол заклинения.

Для проверки принципов работы электромагнитного вариатора был разработан макетный образец, представленный на рис. 17. Все возмущающие воздействия были зафиксированы с помощью осциллографов, настроенных на выходное напряжение тахогенераторов, измеряющих скорость вращения каждого ротора вариатора. На высокой стороне был установлен двигатель постоянного тока, имитировавший ветровую турбину, на низкой стороне был размещен динамический тормоз, имитирующий управление электромагнитного вариатора с изменяемой нагрузкой. Также с его помощью было реализовано возмущающее воздействие. 


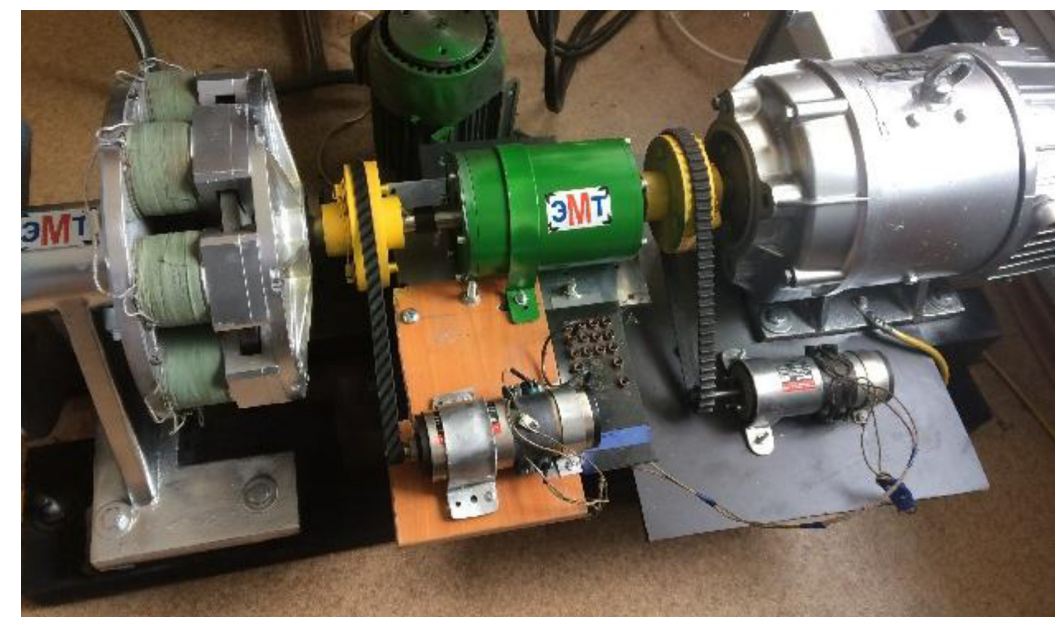

Рис. 17. Макетный образец электромагнитного вариатора

Fig. 17. Model model of an electromagnetic CVT

Компенсация вращающего момента вариатора возможна при помощи контура управления тихоходного ротора турбины. Таким образом, подтверждено экспериментально, что приведенный угол дополнительного рассогласования можно измерять косвенным способом путем известных значений скоростей вращения турбины и генератора, а регулирование этого угла позволяет стабилизировать частоту вращения генератора изменением добавочного момента на валу генератора электромагнитным вариатором.

На рис. 18 отражена снятая механическая характеристика вращающего момента в зависимости от скорости вращения приводного движителя. Результаты теоретических исследований полностью коррелируют с результатами экспериментальных исследований.

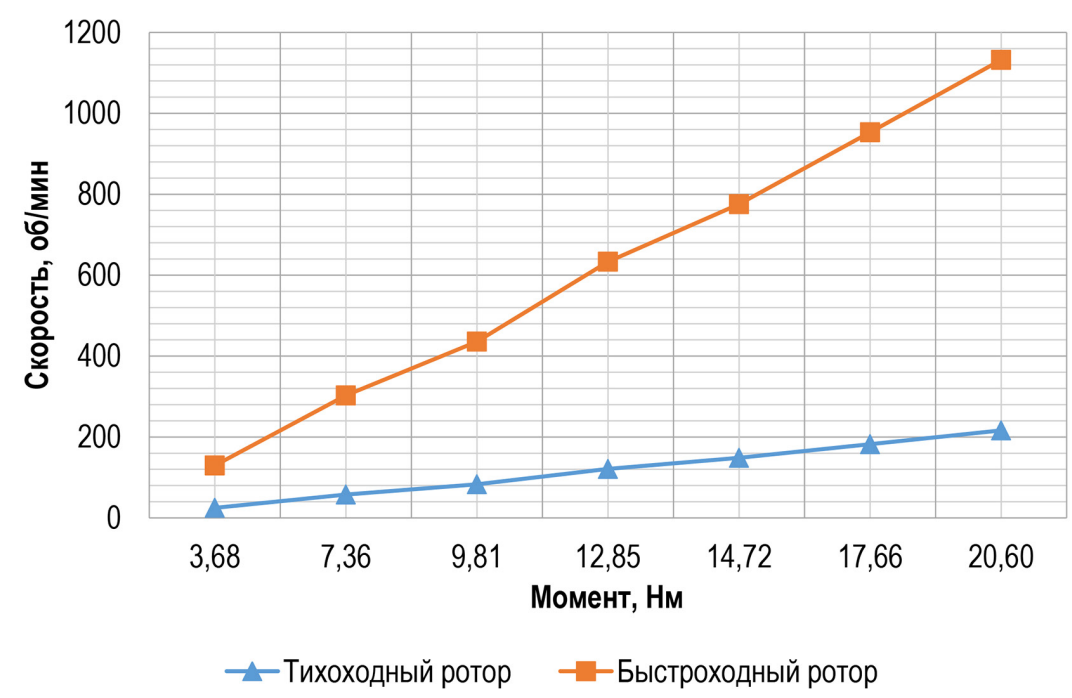

Рис. 18. Механическая характеристика редуктора, полученная экспериментально

Fig. 18. Mechanical characteristics of the gearbox obtained experimentally

$$
-88-
$$




\section{В. Результаты математического моделирования}

Представлены осциллограммы электромеханического переходного процесса при использовании алгоритма стабилизации скорости вращения ротора электрического генератора путем применения комплексного управления электромагнитным вариатором. В случае резкого изменения мощности в энергосистеме при использовании алгоритмов стабилизации скорости вращения магнитного вариатора со стороны генератора наблюдается стабилизация напряжения. Выбор параметров регулятора скорости магнитного вариатора сводился к тому, что решение дифференциального уравнения параллельной работы СГПМ представляет собой апериодический вид без возникновения колебательных процессов. При анализе осциллограмм наблюдается восстановление напряжения после переходного процесса до устранившегося значения [6].

Анализ осциллограмм напряжения и тока, изображенных на рис. 19, показывает, что соблюдается режим генератора до и после переходного процесса. Благодаря действию алгоритма стабилизации скорости сохраняется синхронная работа генератора ВЭУ с энергосистемой.

На рис. 19 представлены осциллограммы скорости вращения и угла положения ротора генератора при стабилизации скорости. В случае отсутствия регулятора скорости при сбросе мощности нагрузки на 15 \% наблюдается увеличение скорости, которое может привести к повреждению генераторов. Алгоритм стабилизации скорости реализует возможность обеспечения синхронной работы генератора и не вызывает значительных механических перегрузок. В режиме резкого сброса нагрузки на 15 \% мощность энергопотребления после переходного процесса снижена в соответствии с уровнем изменения мощности со стороны нагрузки.

Наиболее тяжелым режимом считается трехфазное КЗ. В период КЗ наблюдается снижение напряжения. Возможности магнитного вариатора также предполагают использование алгоритма стабилизации скорости в режиме КЗ [18].

После ликвидации КЗ алгоритм стабилизации скорости формирует управляющее воздействие, обеспечивающее возвращение синхронной скорости вращения после аварии (рис. 20).

Возможно возникновение резкого изменения скорости ветра на ВЭУ. На рис. 21 представлен график скорости ветра на каждой из ВЭУ при рассогласованном его изменении. Отметим, что на первой турбине скорость изменилась с 7 до 15 м/с, а на второй - от 7 до 19 м/с в разные моменты времени [19].

Как упоминалось выше, когда скорость ветра находится в зоне 2, угол заклинения лопасти постоянно поддерживается на оптимальном значении $\beta_{0}$. В этой зоне скорость ротора изменяется пропорционально скорости ветра таким образом, чтобы сохранить соотношение скорости конца лопасти при постоянном значении быстроходности $\lambda_{0}$. В зоне 3 угол заклинения варьируется с целью регулирования скорости ротора генератора и поддержании крутящего момента и мощности на номинальных значениях.

На рис. 22 изображены сравнительные осциллограммы скорости вращения ротора генератора и угла положения ротора при различных изменениях скорости ветра. В реальных ВЭУ не существует систем без регулирования скорости вращения ротора генератора. Поэтому такие режимы не могут существовать на практике. Отметим также, что в случае использования алгоритма стабилизации скорости вращения ротора очень быстро восстанавливается установившийся режим. 
Journal of Siberian Federal University. Engineering \& Technologies 2021 14(1): 72-96
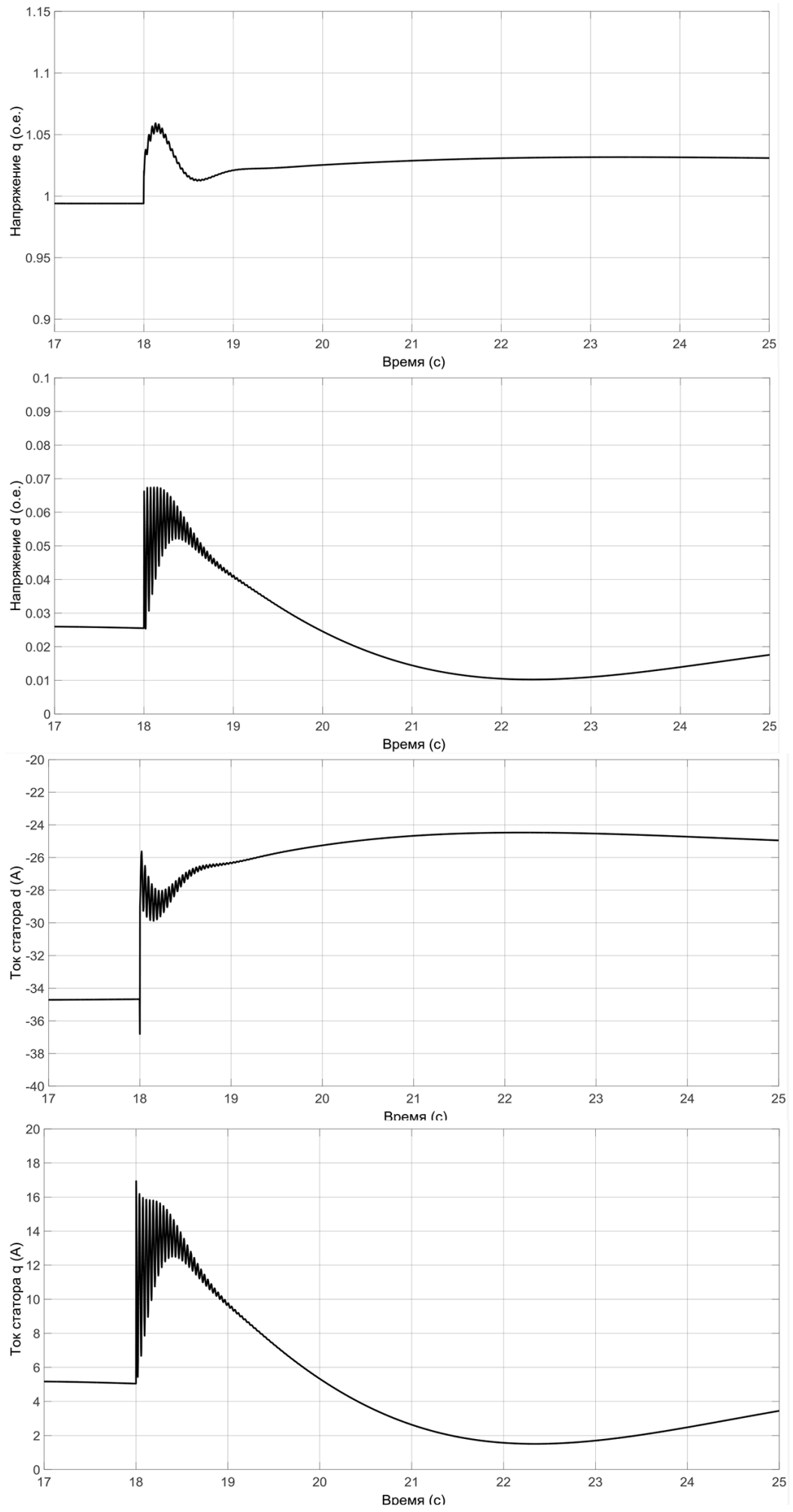

$-90-$ 

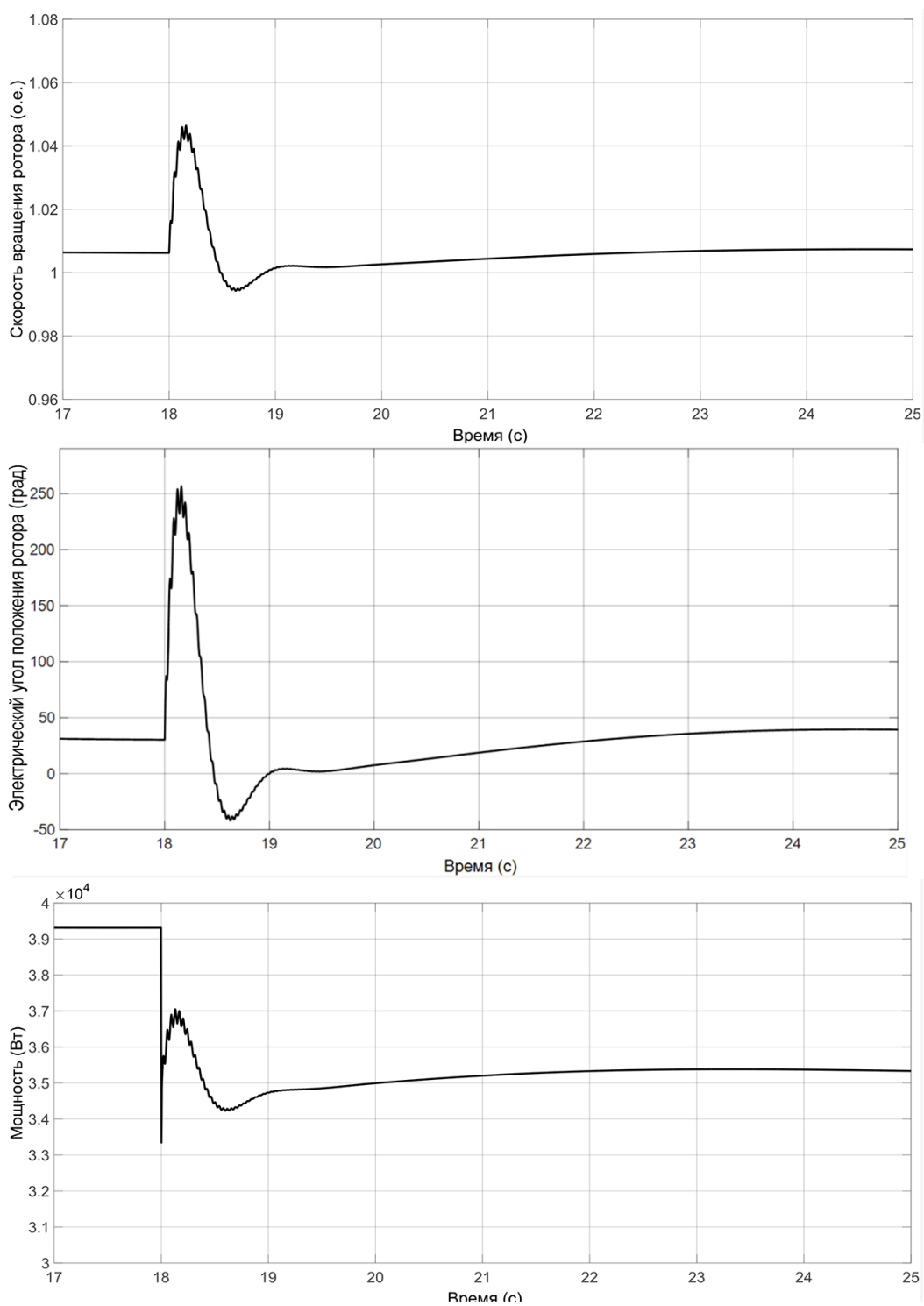

Рис. 19. Осциллограммы напряжения, тока, скорости вращения ротора, угла положения ротора и мощности генератора при использовании алгоритма стабилизации скорости магнитного вариатора при резком снижении мощности на $15 \%$

Fig. 19. Waveforms of voltage, current, rotor speed, rotor position angle and generator power when using the algorithm for stabilizing the speed of the magnetic CVT with a sharp decrease in power by $15 \%$ 

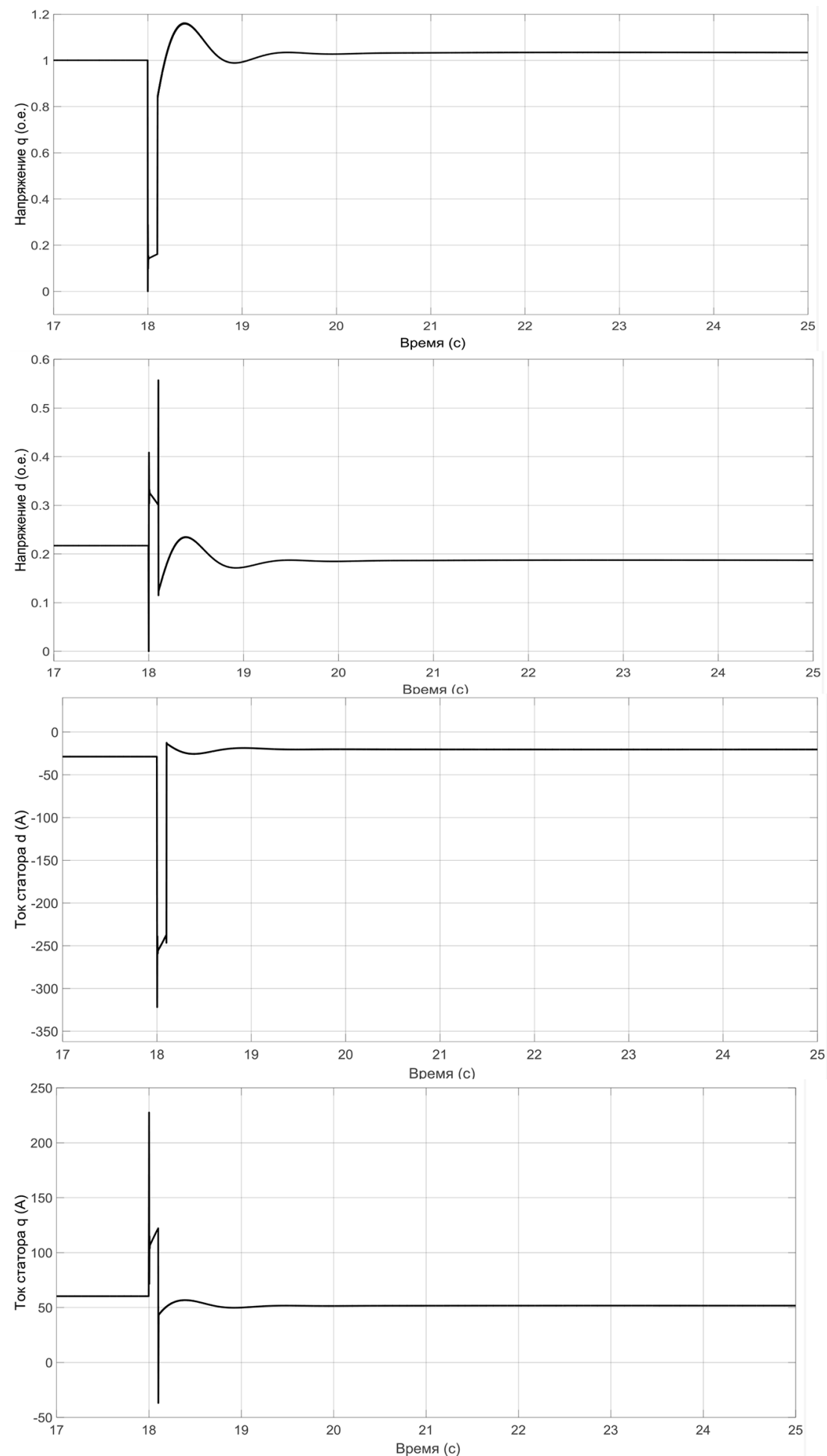

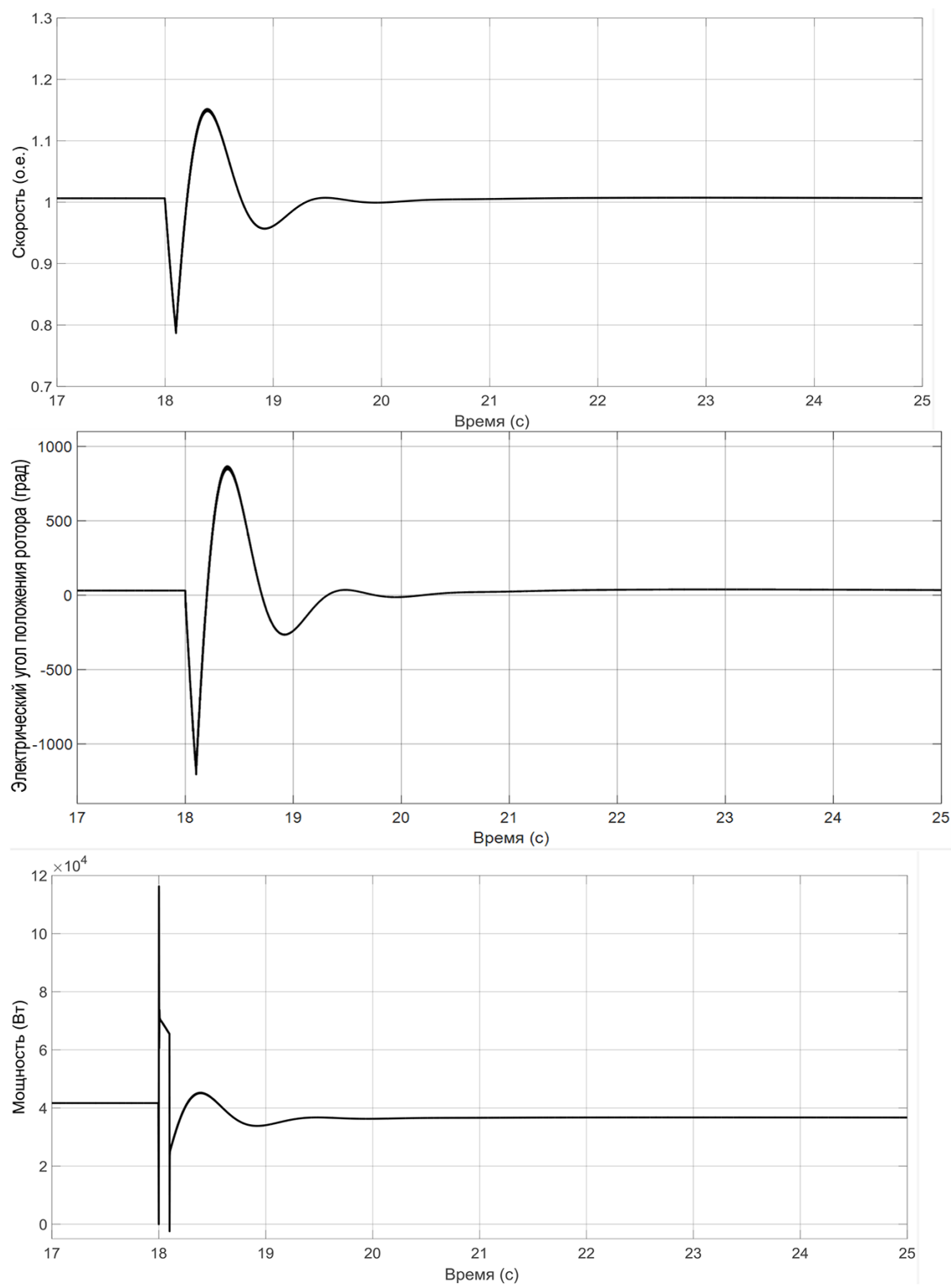

Рис. 20. Осциллограммы напряжения, тока, скорости вращения ротора, угла положения ротора и мощности генератора при использовании алгоритма стабилизации скорости магнитного вариатора при удаленном КЗ

Fig. 20. Waveforms of voltage, current, rotor speed, rotor position angle, and generator power when using the magnetic CVT speed stabilization algorithm with remote short-circuit 


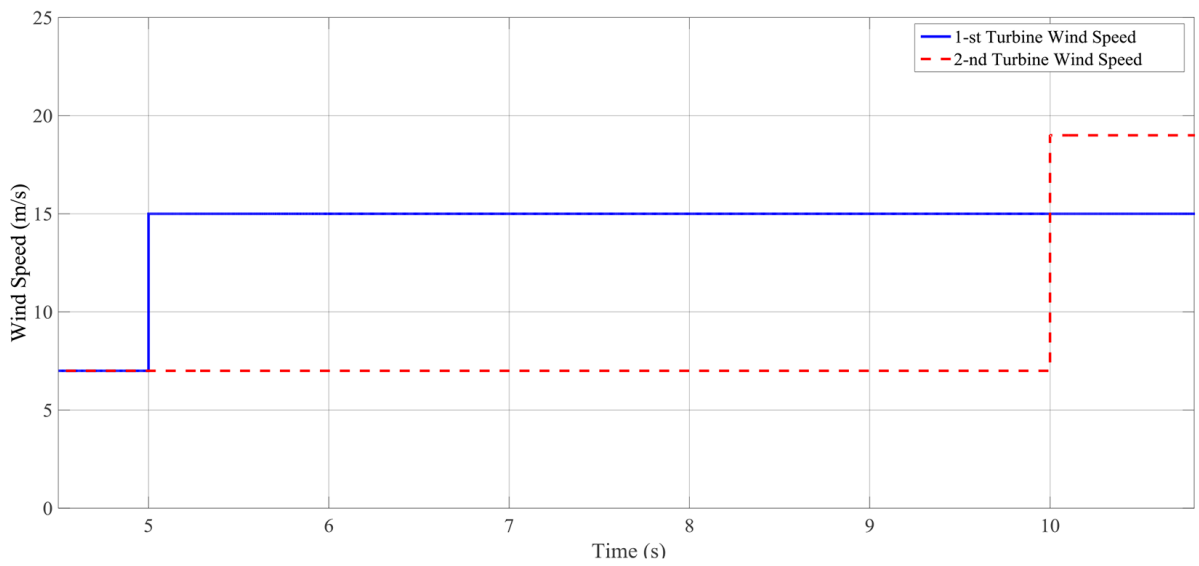

Рис. 21. График изменения скорости ветра на каждой ВЭУ

Fig. 21. Graph of wind speed changes on each wind turbine
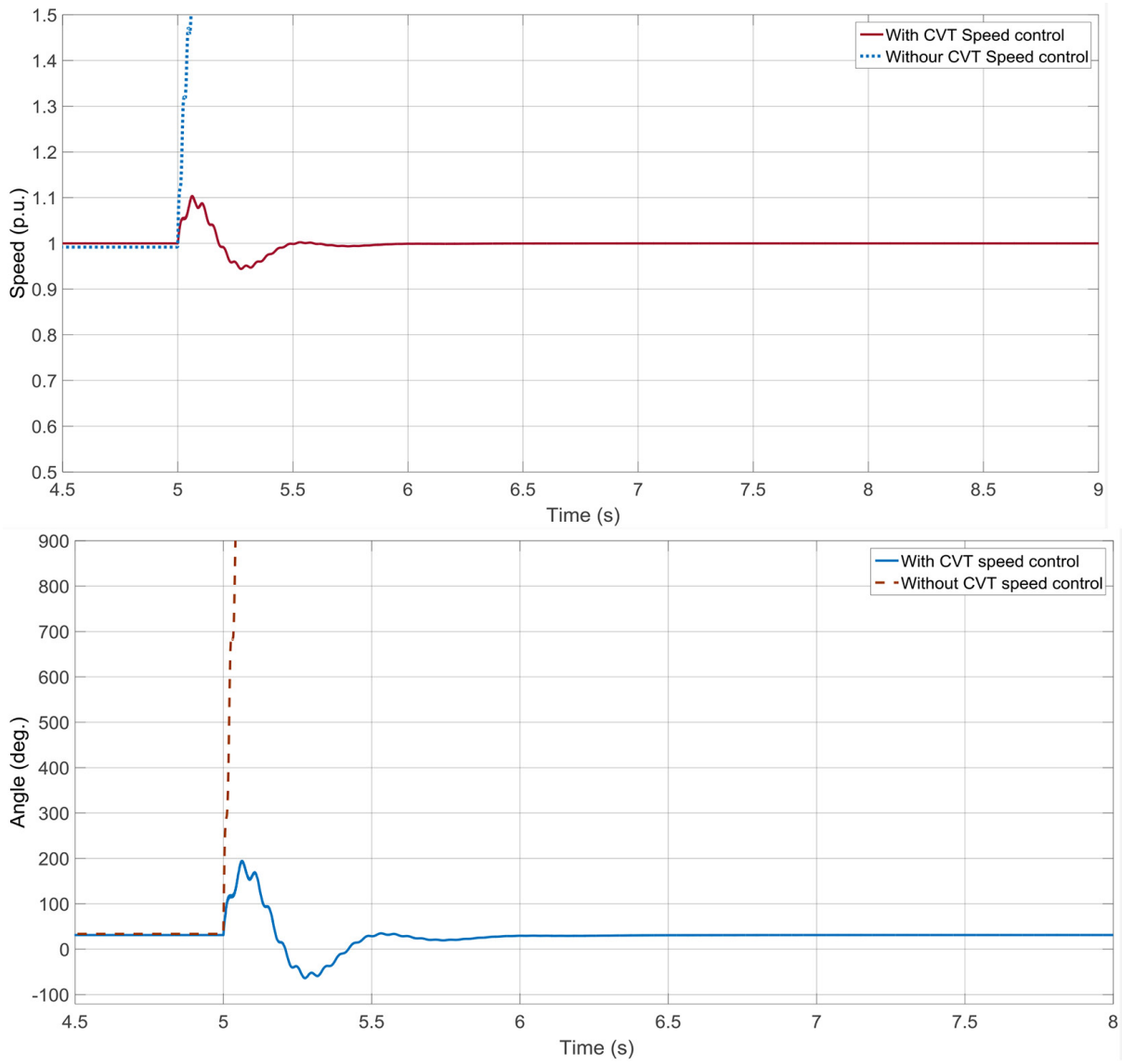

Рис. 22. Осциллограммы скорости и фазового положения ротора генератора при изменении скорости ветра

Fig. 22. Oscillograms of the speed and phase position of the generator rotor when the wind speed changes 


\section{Заключение}

В данной статье исследовалась работа контроллера комплексного управления с оценкой скорости ветра онлайн для ветровой турбины. Предложена схема, состоящая из магнитного вариатора и системы управления стабилизации скорости вращения его выходного ротора. Численные расчеты при резком изменении нагрузки на 15 \% и КЗ показали восстановление параллельной работы СГПМ через 10 с. Исследован подход к сопряжению генератора ВЭУ с магнитным вариатором в составе энергетической системы без использования вставки постоянного тока в силовой части цепи.

\section{Список литературы / References}

[1] E.A.Bossanyi. The design of closed loop controllers for wind turbines. Wind energy, 2000, 3, $148-163$

[2] K.Z.Ostergaard, P. Brath, J. Stoustrup. Gain-scheduling linear quadratic control of wind turbines operating at high wind speed. IEEE International Conference on Control Applications, 2007, $276-281$

[3] K.Stol, M. Balas. Full-state feedback and constant gains. ASME J. Solar energy engineering, 2001, 123(4), 319-326

[4] E.B.Muhando, T. Senjyu, H. Kinjo, T. Funabashi. Augmented LQG controller for enhancement of oline dynamic performance for WTG system. Renewable energy, 2008, 33, 1942-1952

[5] Удалов С.Н., Манусов В.З. Моделирование ветроэнергетических установок и управление ими на основе нечеткой логики. Новосибирск, НГТУ, 2013, 200. [Udalov S.N., Manusov V.Z. Modeling of wind power facilities and their management on the basis of fuzzy logic. Novosibirsk, NGTU, 2013, 200 (in Russian)]

[6] Sergey N. Udalov, Andrey A. Achitaev, Alexander G. Pristup, Boris M. Bochenkov and Richard D. Tarbill. Increasing the Regulating Ability of a Wind Turbine in a Local Power System Using by Magnetic Continuous Variable Transmission. Wind Engineering, 2018, 42(5), $411-435$

[7] Sapsalev A. V. et al. Structural model of a magnetic gearbox. 18th International Conference of Young Specialists on. IEEE, 2017, 568-571

[8] Montague R., Bingham C., Atallah K. Servo control of magnetic gears. IEEE/Asme Transactions on Mechatronics. 2012, 17(2). 269-278

[9] Jafari, Seyyed Hosein, Mahdi Raoofat, and Haidar Samet. Improving transient stability of double fed induction generator using fuzzy controller. International Transactions on Electrical Energy Systems. 2014, 1065-1075.

[10] E.L. van der Hooft, P. Shaak, T.G. van Engelen. Wind turbine control algorithms. ECN-C-03-111, 2003.

[11] Udalov S. N., Achitaev A. A., Pristup A. G., Bochenkov B. M. Improving dynamic stability of a wind turbine using a magnetic continuously variable transmission. IEEE, 2016, 1-4

[12] K. Ogata, Modern Control Engineering. Upper Saddle River, New Jersey: Prentice-Hall, $2002,956 \mathrm{p}$.

[13] G. Zhang and J. Furusho. Speed control of two-inertia system by PI/PID control. Power Electronics and Drive Systems. 1999, 1, 567-572. 
[14] T. M. O'Sullivan, C. M. Bingham, and N. Schofield. High-performance control of dual-inertia servodrive systems using low-cost integrated SAW torque transducers. Industrial Electronics, 2006, $53,1226-1237$

[15] Удалов С. Н., Ачитаев А.А., Приступ А.Г., Боченков Б. М. Повышение регулировочной способности ветроэнергетической установки в составе локальной энергосистемы. Энергобезопасность и энергосбережение, 2017, 3, 33-40. [Udalov S. N., Achitaev A.A., Pristup A. G., Bochenkov B. M. Increasing the regulating ability of a wind power plant in the structure of the power system. Energy security and energy saving., 2017, 3, 33-40 (in Russian)]

[16] Удалов С.Н. Ачитаев А.А., Приступ А.Г., Топорков Д.М. Повышение эффективности ветроэнергетической установки путем использования псевдопрямого привода. Энергобезопасность и энергосбережение. 2017, 5, 59-63. [Udalov S.N., Achitaev A.A., Pristup A.G., Toporkov D. M. Improving the efficiency of a wind turbine by using a pseudo-direct drive. Energy security and energy saving, 2017, 5, 59-63 (in Russian)]

[17] Atallah K., Wang J. A brushless permanent magnet machine with integrated differential. IEEE Transactions on Magnetics, 2011, 47(10), 4246-4249

[18] Hinrichsen E. N., Nolan P. J. Dynamics and stability of wind turbine generators. IEEE Transactions on Power Apparatus and Systems, 1982, 8, 2640-2648

[19] Udalov S. N., Achitaev A.A., Pristup A.G., Bochenkov B.M., Pankratc Y.V. Using a Magnetic Continuously Variable Transmission for Synchronization of Wind Turbine Generators Under a Variable Wind Speed. Dynamics of Systems, Mechanisms and Machines (Dynamics). 2017, 1-5 